\title{
"Vegetación de un bosque de Polylepis incarum (Rosaceae) en el distrito de Lampa, Puno, Perú": Un comentario sobre la Fitosociología en el Perú
}

\section{"Vegetation of a Polylepis incarum forest (Rosaceae) in Lampa district, Puno, Peru": Comment on Phytosociology in Peru}

\section{Antonio Galán de Mera}

Laboratorio de Botánica, Universidad CEU San Pablo, Apartado 67, 28660 Boadilla del Monte, Madrid, España.

Email Antonio Galán de Mera: agalmer@ceu.es

\section{Sr. Editor,}

Hace un tiempo pude leer en la Revista Peruana de Biología [22(1): 87-96 (2015) DOI: http://dx.doi.org/10.15381/rpb. v22i1.11125] el trabajo titulado "Vegetación de un bosque de Polylepis incarum (Rosaceae) en el distrito de Lampa, Puno, Perú", donde sorpresivamente aparece mi nombre en "agradecimientos" por mis comentarios fitosociológicos a los autores (Montesinos-Tubée, Pinto, Beltrán \& Galiano). Aunque ciertamente, hace un año, el primer autor me preguntó sobre la validez y nomenclatura de la alianza fitosociológica Ribesido brachybotrys-Polylepidion besseri Galán de Mera \& Cáceres (Galán de Mera et al. 2002), y a lo que yo respondí diciendo que el nombre de la alianza habría que corregirlo (Email de 23/04/2014), me asombra que mi respuesta haya tenido algo que ver en los comentarios realizados a este trabajo, pues nunca los hice. Tampoco me gustaría que mi nombre quedase unido a esta investigación por razones profesionales y por respeto al resto de los botánicos del Perú que conocen las investigaciones que realizo con mi equipo desde 1987, puesto que yo no puedo avalar gran parte de lo que ahí se dice desde el punto de vista fitosociológico.

Las razones profesionales, claro está, dentro del campo de la Fitosociología, son las siguientes si tomamos como base la bibliografía de este campo de la Botánica y los artículos (art.) del Código Internacional de Nomenclatura Fitosociológica traducidos al español para evitar errores de interpretación (Izco \& Del Arco 2003):

El nombre Ribesido brachybotrys-Polylepidion besseri no ha sido corregido (art. 43) de la forma que yo mismo indiqué al primer autor. La nomenclatura y autorías correctas deberían ser:
Ribesido brachybotrys-Polylepidion rugulosae Galán de Mera \& Cáceres in Galán de Mera, Rosa \& Cáceres, nom. corr. hoc loco [Ribesido brachybotrys-Polylepidion besseri Galán de Mera \& Cáceres in Galán de Mera, Rosa \& Cáceres, in Acta Bot. Malacitana 27: 87 (2002)], puesto que Polylepis besseri Hieron. no es la especie que alcanza los Andes del SW de Perú, sino $P$. rugulosa (Kessler \& Schmidt-Lebuhn 2006).

En "Materiales y métodos", los autores dicen que "los cuadrantes fueron seleccionados de acuerdo a la homogeneidad de la vegetación”, cuando posteriormente en la Tab. 3 existe una mezcla de plantas características que nos están indicando todo lo contrario, lo cual es contradictorio con la metodología de la escuela de Zürich-Montpellier (Braun-Blanquet 1979). Cabría preguntar a los autores que cuál es el criterio para definir dichos cuadrantes, puesto que un poco más adelante, en el mismo párrafo, indican que "los cuadrantes fueron establecidos con una dimensión de $225 \mathrm{~m}^{2}$ basando el tamaño aplicado para la vegetación arbórea-arbustiva con pajonales...”, lo cual predice la mezcla de especies entre clases fitosociológicas y el distanciamiento respecto a asociaciones (de árboles, arbustos, matorrales y pastizales) que definen el dinamismo de la vegetación (Géhu \& Rivas-Martínez 1981, Dengler et al. 2008).

La asociación descrita no corresponde a la alianza Ribesido brachybotrys-Polylepidion rugulosae, puesto que esta es de la puna seca (provincia biogeográfica Oruro-Arequipa)(Galán de Mera et al. 2003, Luebert \& Gajardo 2005), y la cuenca del Titicaca corresponde a la provincia biogeográfica (Ancash-La Paz). Esto queda además demostrado con la última aproximación sistemática del género Polylepis en el Perú (Mendoza \& Cano 2012).

$\begin{array}{ll}\text { Presentado: } & 22 / 06 / 2015 \\ \text { Aceptado: } & \\ \text { Publicado online: } & 14 / 10 / 2015\end{array}$

\section{Citación:}

Galán de Mera A. 2015. "Vegetación de un bosque de Polylepis incarum (Rosaceae) en el distrito de Lampa, Puno, Perú": Un comentario sobre la Fitosociología en el Perú. Revista peruana de biología 22(2): 263 - 264 (Octubre 2015). doi: http://dx.doi.org/10.15381/rpb.v22i2.11361 
La asociación Lupino chlorolepis-Polylepidetum incari [errore, recte incarum] es inválida, puesto que presenta el mismo holótipo que la subasociación vicietosum gramineae (art. 5), y por tanto, el resto de las subasociaciones también son inválidas (art. 4). Además, si observamos la Tab. 3, no hay diferencias florísticas entre vicietosum gramineae y Lupino-Polylepidetum, y sus especies diferenciales solo manifiestan una intervención antrópica que indica que estamos ante una comunidad derivada (Kopecký et al. 1995, Schaminée \& Stortelder 1996), sobre todo por la presencia de Stipa ichu (Seibert \& Menhofer 1992).

Es similar lo que ocurre con la subasociación solanoetosum [errore, recte solanetosum] nitidae [errore, recte nitidi], aunque aquí ni siquiera están claras las plantas características, pues los autores primero escriben sobre especies de elevada frecuencia, luego sobre diagnósticas (donde no está Solanum nitidum), y luego sobre especies diferenciales que no indican la misma situación ecológica en otras localidades del Perú.

Aunque las fotografías son extraordinarias, no se logra entender cómo las Figs. 3 y 4 puedan tratarse de un bosque.

Realmente es halagador y enriquecedor que los autores empleen la metodología de los pisos bioclimáticos para definir cinturas de vegetación, y de que traten de generar nuevas perspectivas dentro de la ya longeva Fitosociología peruana, iniciada en el seno de las facultades de Farmacia y Biología de la Universidad Nacional Mayor de San Marcos (López Guillén \& Rivas Goday 1952, Gutte 1978, Rivas-Martínez \& Tovar 1982). Sin embargo, hay que tener cuidado con el empleo superfluo del nombre de otros autores. Debemos tener en cuenta que la Fitosociología no puede ser subjetiva ni en el levantamiento de inventarios en el campo ni en el uso de herramientas informáticas, y que la definición y nomenclatura de los sintáxones debe quedar sujeta al Código. Solo así podremos llevarla fuera de las fronteras peruanas interaccionando con los colegas que describen unidades de vegetación próximas a las nuestras [Colombia: Rangel et al. 1997, Bolivia: Navarro \& Maldonado 2005, Chile: Luebert \& Pliscoff 2006, Argentina: Méndez 2011, Ecuador: Izco 2013, Venezuela: Galán de Mera 2014].

Sr. Editor, en no pocos países la Fitosociología es utilizada para cartografiar unidades de vegetación y proceder a la gestión de sus territorios. Si en el Perú no tenemos la responsabilidad de aplicarla con objetividad, caerá en vano todo el trabajo investigador y docente realizado durante muchos ańos, ahora que ya comienzan a utilizar sus resultados desde empresas de consultoría ambiental a gobiernos regionales.

\section{Literatura citada}

Braun-Blanquet J. 1979. Fitosociología. H. Blume. Madrid.

Dengler J., M. Chytrý \& J. Ewald. 2008. Phytosociology. En: Jørgensen S.E. \& B.D. Fath (Eds.) Encyclopedia of Ecology. Elsevier. Oxford. pp: 2767-2779.

Galán de Mera A. 2014. La vegetación de las sabanas de los Llanos de Venezuela. En: Rangel J.O. (Ed.) La región de la Orinoquia de Colombia. Colombia Diversidad Biótica XIV. Universidad Nacional de Colombia. Bogotá. pp.: 447-482.

Galán de Mera A., M.V. Rosa \& C. Cáceres. 2002. Una aproximación sintaxonómica sobre la vegetación del Perú. Clases, órdenes y alianzas. Acta Botanica Malacitana 27: 75-103.

Galán de Mera A., C. Cáceres \& A. González. 2003. La vegetación de la alta montaña andina del sur del Perú. Acta Botanica Malacitana 28: 121-147.

Géhu J.M. \& S. Rivas-Martínez. 1981. Notions fondamentales de Phytosociologie. En: Dierschke H. (Ed.) Syntaxonomie. J. Cramer. Vaduz. pp.: 5-33.

Gutte P. 1978. Beitrag zur Kenntnis zentralperuanischer Pflanzengesellschaften I. Ruderalpflanzengesellschaften von Lima und Huanuco. Feddes Repertorium 89: 75-97.

Izco J. 2013. The Biological Reserve of San Francisco (Ecuador): Revision of the syntaxonomy and nomenclature of the vegetation. Lazaroa 34: 257-266.

Izco J. \& M. Del Arco. 2003. Código internacional de nomenclatura fitosociológica. Universidad de La Laguna.

Kessler M. \& A.N. Schmidt-Lebuhn. 2006. Taxonomical and distributional notes on Polylepis (Rosaceae). Organisms, Diversity and Evolution 5 (Electr. Suppl. 13): 1-10.

Kopecký K., J. Dostalek \& T. Frantik. 1995. The use of the deductive method of syntaxonomic classification in the system of vegetational units of the Braun-Blanquet approach. Vegetatio 117: $95-112$.

López Guillén J. \& S. Rivas Goday. 1952. Preferencias edáficas de la Distichlis spicata (L.) Greene, en los "gramadales" de Conchán (Lima, Perú). Anales del Jardín Botánico de Madrid 10: 605-618.

Luebert F. \& R. Gajardo. 2005. Vegetación alto andina de Parinacota (norte de Chile) y una sinopsis de la vegetación de la Puna meridional. Phytocoenologia 35: 79-128.

Luebert F. \& P. Pliscoff. 2006. Sinopsis bioclimática y vegetacional de Chile. Editorial Universitaria. Santiago de Chile.

Méndez E. 2011. La vegetación de los Altos Andes. El flanco oriental del Cordón del Portillo (Tunuyán, Mendoza, Argentina). Boletín de la Sociedad Argentina de Botánica 46: 317-353.

Mendoza W. \& A. Cano. 2012. El género Polylepis en el Perú. Taxonomía, morfología y distribución. Editorial Académica Española. Saarbrücken.

Navarro G. \& M. Maldonado. 2005. Geografía ecológica de Bolivia. Vegetación y ambientes acuáticos. Fundación Simón I. Patińo. Santa Cruz.

Rangel J.O. 1997. Colombia Diversidad Biótica II. Tipos de vegetación en Colombia. Universidad Nacional de Colombia. Bogotá.

Rivas-Martínez S. \& O. Tovar. 1982. Vegetatio Andinae, I. Datos sobre las comunidades vegetales altoandinas de los Andes Centrales del Perú. Lazaroa 4: 167-187.

Schaminée J.H.J. \& A.H.F. Stortelder. 1996. Recent developments in phytosociology. Acta Botanica Neerlandica 45: 443-459.

Seibert P. \& X. Menhofer. 1992. Die Vegetation des Wohngebietes der Kallawaya und des Hochlandes von Ulla-Ulla in den bolivianischen Anden, Teil II. Phytocoenologia 20(3): 289-438. 